\title{
A New Technique for Location of Fault Location on Transmission Lines
}

\author{
Khalaf Y. Alzyoud \\ Al- Balqa' Applied University - Jordan \\ Amman, P.O.Box (15008), marka ashamalia \\ E-mail: yasin_mualla@yahoo.com
}

Al-Mofleh Anwar (Corresponding author)

School of Electrical \& Electronic Engineering, Universiti Sains Malaysia, Pulau Pinang

E-mail: anwaralmofleh@yahoo.com

Faisal Y. Alzyoud

School of Computer Science, Universiti Sains Malaysia, Pulau Pinang

E-mail: zoiod_jo@yahoo.com

\begin{abstract}
Magnetic field sensors can be used to detect fault detection and location effectively. Quick fault detection can help protect equipment by allowing the disconnection of faulted lines before any significant damage is done. A variety of algorithms continue to be developed to perform this task more accurately and more effectively. Particularly fault impedance (based algorithms) require both current and voltage information. However, it is possible to monitor a transmission system without using current or voltage transformers through the analysis of the magnetic field near the conductors. Since each conductor in a transmission line creates a magnetic field due to the current pass through it. Magnetic field sensors are used and tested using MATLAB through analyzing wave fault detection and location as there is a possibility of analyzing the transmission line system based on the resultant magnetic field produced. The results show that the magnetic field sensors are a viable tool for power transmission line fault detection, so this method is highly recommended to be used based on its efficient and accuracy.
\end{abstract}

Keywords: Transmission line, Fault detection, Fault location, Travelling waves, Current transformers

\section{Introduction}

Transmission line is one of the main components in every electric power system. The transmission line is exposed to the environment and the possibility of experiencing faults on the transmission lines ( Bouthiba, 2004) ( Salama, 1999). Line faults are the most common faults, they may be triggered by lightning strokes, trees may fall across lines, fog and salt spray on dirty insulators may cause the insulator strings to flash over, and ice and snow loadings may cause insulator strings to fail mechanically (Tziouvaras et al., 2001) ( PES, 2004). When a fault occurs on an electrical transmission line, it is very important to detect it and to find its location in order to make necessary repairs and to restore power as soon as possible (Firouzjah and Sheikholeslami ,2009). As the time needed to determine the fault point along the line will reflect the quality of the power delivery. Therefore, an accurate fault location on the line is an important requirement for a permanent fault. Pointing to a weak spot, it is also helpful for a transient fault, which may result from a marginally contaminated insulator, or a swaying or growing tree under the line (Zimmerman and Costello, 2005). Due to the simple relationship between current and magnetic field intensity, it is understandable that magnetic field sensors have previously been used in fault detection and location schemes. These schemes often use magnetic field sensors in place of current transformers since magnetic field sensors can be installed independently from a substation or switching station with a minimum amount of additional equipment ( Sneddonand and Gale, 1997)(Aurangzeb et al., 2000). One possible use of this relationship is simply replacing each current transformer with a Hall Effect transducer. This transducer would typically need to be within the electrical arcing distance of the conductors to produce enough voltage for analysis and would thus require insulation (Khorashadi-Zadeh and Sanaye-Pasand, 2006). To remove this need for insulation, the transducer can be located between two tapered pieces of ferromagnetic material in order to concentrate the magnetic field into the transducer; as a result, the transducer does not need to be located within the arcing distance of the conductors. The measured magnetic field result can then be used similarly as a 
current measurement for fault detection and location (Elhaffar and Lehtonen, 2004) (Vintan, 2004). Since a magnetic field sensor does not need to make contact with the conductors and can be installed remotely from substations, the effectiveness of the magnetic field sensor in fault detection and location algorithms is clearly worth examining (Jiang et al., 2000).

\section{Conductor Configurations and the Magnetic Field}

The magnetic field which is detected by the current sensors will be changed based on the configuration of the conductors. Since the magnetic field-based fault detector should be able to be used in a variety of situations, it is essential to gain an understanding of its performance for several conductor configurations (PSRC, 1999). The magnetic fields of five different conductor configurations are demonstrated below in Figures. Each Figure shows the conductor configuration, the magnetic field during balanced operation, the magnetic field during a single line to ground fault on phase $a$, and the magnetic field during a line to line fault on phases $a$ and $b$. The currents in the un faulted conductors are assumed to have an RMS value of 100A; the fault currents are assumed 1000A RMS. The value of (P) was set as 2 meters, and the conductors are assumed to correspond to phases $(a, b$, and $c)$ from left to right. Figure 1 shows a horizontal conductor configuration and its magnetic fields. The sensors are located a distance $\mathrm{p}$ below phase $b$ where $\mathrm{p}$ is the distance between phases ( $a$ and $c$ ). As expected, the vertical component of the magnetic field during normal operation is greater than the horizontal component. The vertical maximums occur when the current in phase $b$ is crossing zero and as a result, the other phases cancel each others' horizontal components but add them in the same direction vertically. Likewise, the horizontal maximums occur when the current in phase $(b)$ is a maximum; however, at this time the horizontal components of the magnetic fields due to phases $(a)$ and $(c)$ add in the opposite direction and cancel some of the horizontal magnetic field due to phase $(b)$, while the vertical components due to phases $(a)$ and $(c)$ cancel each other. The magnetic field of the single line to ground fault on phase $(a)$ as expected. The magnetic field contributions due to phases $(b)$ and $(c)$ become relatively insignificant, so the magnetic field is essentially only the field due to phase $(a)$. The angle of rotation is also mostly determined by the angle between the phase a conductor and the sensors from a vertical reference. Neglecting the effects of the other phases, the angle of rotation would be:

$$
\tan ^{-1}\left(\frac{p / 2}{p}\right)=\tan ^{-1}(0.5) \approx 26.6^{\circ}
$$

Which is fairly close to the observed angle of rotation, the difference is due to the fact that the calculated angle does not account for the currents in phase's $(b)$ and $(c)$. The line to line fault on phases $(a)$ and $(b)$ creates a magnetic field which is somewhat unexpected, particularly with respect to the angle of rotation. Since the magnitudes of the currents in phases $a$ and $b$ are identical in this situation and they are approximately $180^{\circ}$ out of phase due to the line to line fault, the positive maximum horizontal field due to phase $a$ occurs when the current is at its positive maximum, so it is counteracted by a field inclined at the same angle as noted above. As a result, this maximum magnetic field has a positive horizontal component and a negative vertical component. The same result in the opposite direction occurs when the current in phase $a$ is at its negative maximum and phase $b$ is at its positive maximum. It is clear from these plots that, at least for a significant fault current, a line to ground or line to line fault could be detected using magnetic sensors for this conductor configuration. The conductor configuration shown in Figure 2 is a delta configuration. The magnetic fields here are similar to those of the horizontal conductor configuration under normal operating conditions and for the single line to ground fault on phase $a$. However, the line to line fault on phases ( $a$ and $b$ ) has a very different angle of rotation. The reason for this is that the magnetic field is inversely proportional to the square of the distance from the conductor; the contribution from phase $b$ is significantly reduced compared to its contribution for the case of the horizontal conductor configuration. As a result the analysis above regarding the horizontal configuration remains true; the magnitude of the maximum magnetic field due to phase $b$ is reduced. Thus, the magnetic field is more significantly affected by the current in phase $(a)$, so the maximum positive horizontal component due to phase $b$ is less than the negative horizontal component due to phase (a) concurrently. This in turn causes the significant change in the angle of rotation of the ellipse.

\section{Location and Basic Design of the Sensors}

By performing this analysis in polar coordinates rather than Cartesian coordinates, the fault detection is based on two variables (rho and theta), but in essence only one of these is used per algorithm. This makes fault detection more intuitive, since only one time-dependent variable needs to be analyzed for each algorithm rather than the three (or more) variables in a typical fault detection and location scheme. The values of rho and theta are given by equation 2,3 . 


$$
\begin{aligned}
& \rho=\sqrt{H_{X}^{2}}+H_{Y}^{2} \\
& \theta=\tan ^{-1}\left(\frac{H_{Y}}{H_{X}}\right)
\end{aligned}
$$

Where the result of the inverse tangent has been corrected if $\left(\mathrm{H}_{\mathrm{X}}\right)$ is negative

Assuming a triangular or horizontally coplanar arrangement of conductors, the sensors should ideally be located fairly close to the transmission lines vertically to reduce the effects of magnetic interference, since magnetic interference can affect the accuracy of fault detection and in extreme cases to indicate faults where they do not occur. Also, keeping the sensors fairly close to the transmission lines helps in keeping the shape of the magnetic field intensity close to a circle as possible. The shape of the magnetic field intensity ellipse can also cause problems in detection since long, narrow ellipses increase the likelihood of incorrect fault detection. While the magnetic field ellipse could be kept close to a circle in shape in the processing algorithm, it is much more difficult to reduce or eliminate magnetic interference. It should be noted that the close proximity to the conductors improves the performance of the analysis as stated above; the sensors must be kept outside the arcing distance of any of the conductors. Additionally, the sensors should be horizontally located directly under the center phase (if possible) to create an elliptical rotating magnetic field that is close to a circle as possible and it has an angle of rotation that is very close to $0^{\circ}$ or $90^{\circ}$, this is to reduce incorrect fault detection. The location of the sensors relative to the conductors and the resulting magnetic field are shown below in Figure 3. As the sensors are shifted horizontally, the angle of rotation of the elliptical rotating magnetic field begins to increase in the direction shown; because when the horizontal magnetic field due to phase $c$ is at its positive maximum, the currents in phases $a$ and $b$ contribute to the magnetic field negatively, both in the horizontal and vertical directions. This negative contribution in the horizontal direction is not enough to overcome the contribution due to the current in phase $c$, so this maximum value of the magnetic field is in the fourth quadrant similarly to the current in phase $c$ when it is at its negative maximum. A representative sensor location and the resulting magnetic field are shown in Figure 4. As the sensors are shifted further, the angle of rotation of the magnetic field continues to increase and the magnitudes of the maximums also begin to decrease; the minimums also decrease rapidly. This simplicity is due to the increase of distance from all of the conductors. An example sensor position and the magnetic field are shown in Figure 5. As the sensors are shifted even further, the angle of rotation of the elliptical rotates magnetic field increases beyond $180^{\circ}$ simultaneously, so the maximum value of the magnetic field decreases even more. This rotation is due to the increased percentage of the magnetic field resulting from phase $c$ as well as the fact that the angles that all three phases make with the sensors from vertical are becoming closer in their value. A sensor location which demonstrates this and the corresponding magnetic field are shown in Figure 6. In addition to being located under the center of the transmission line and as close to the conductors as possible, the magnetic field sensors should also be located away from magnetic materials or materials that can be magnetized, since these will affect the magnetic field perceived by the sensors. This means that the sensors needs to be mounted on non-magnetic poles; this is of special note in the case that the pylons used in the transmission system in question are made of material that can be magnetized such as steel. The suggested designs for the magnetic field sensors are two search coils, one oriented such that its induced voltage is proportional to the vertical magnetic field and one such that its induced voltage is proportional to the horizontal magnetic field. The voltages induced in the coils are

$$
\begin{gathered}
V_{X}=\mu_{O} N A \frac{\delta H_{X}}{\delta t}=K \omega H_{X} \cos \left(\omega t+\theta_{X}\right) \\
v_{y}=\mu_{o} N A \frac{\partial H_{y}}{\partial t}=k \omega H_{y} \cos \left(\omega t+\theta_{y}\right)
\end{gathered}
$$

Where the coil constant is

$$
K=\mu_{o} N A
$$

And

\section{Fault Location Analysis}

$$
\mu_{o}=4 \pi \times 10^{-7} \mathrm{H} / \mathrm{m}
$$

For the analyzed system, the length of the transmission line and the velocity of propagation are assumed to be known. This analysis is performed based on two sets of sensors located at opposite ends of the transmission line as shown in Figure 7. Although the velocity of propagation in the transmission line can be described using the 
Telegrapher Equations.

$$
u=\frac{1}{\sqrt{L C}}
$$

For a lossless transmission line, where $u$ is the velocity of propagation and $\mathrm{L}$ and $\mathrm{C}$ are the inductance and capacitance of the transmission line per unit length, respectively, this method of determining the velocity is somewhat cumbersome in this situation; since $\mathrm{L}$ and $\mathrm{C}$ are difficult to be measured for a multi-conductor transmission line. Since the velocity of propagation in a un faulted transmission line, so it can be described as

$$
u=\frac{1}{t_{\text {trans }}}
$$

Where $t_{\text {trans }}$ is the time for an impulse at one end of the transmission line to reach the other end, the velocity of propagation $u$ can be measured without knowing directly the line capacitances and inductances. It is also known that $u$ can be estimated to be close to the speed of light in power transmission and distribution lines; however, an accurate value of the propagation velocity is essential in accurate traveling wave-based fault location. Once $u$ is known, the distance from each sensor and the actual time at which a fault occurs can be found using the equations below

And

$$
d_{1}-d_{2}=u\left(t_{1}-t_{2}\right)
$$

$$
d_{1}+d_{2}=l
$$

Where $t_{1}$ and $t_{2}$ are the times at which faults were detected by each sensor in seconds, $d_{1}$ and $d_{2}$ are the distances from the fault to each sensor in kilometers, $l$ is the length of the transmission line in kilometers, and $u$ is the velocity of propagation in kilometers per second. These result in the following equations:

$$
\begin{aligned}
& d_{1}=\frac{1}{2}\left[l+u\left(t_{1}-t_{2}\right)\right] \\
& d_{2}=\frac{1}{2}\left[l+u\left(t_{2}-t_{1}\right)\right]=l-d_{1} \\
& t_{\text {fault }}=t_{1}-\frac{d_{1}}{u}=t_{2}-\frac{d_{2}}{u}
\end{aligned}
$$

Where $\left(t_{\text {fault }}\right)$ is the estimated time at which the fault actually occurred. The actual fault time $\left(t_{\text {fault }}\right)$ is close to the times at which the fault is detected because $u$ is typically very close to the speed of light. Since the fault detection will be performed by a microprocessor, the analog to digital conversion sampling rate is an important consideration in the accuracy of fault detection. The sampling rate is directly related to the maximum accuracy of the algorithm, since it determines the minimum measurable difference in fault detection times. As a result, the sampling rate must be fairly high to obtain dependable accuracy. The difference in calculated fault location when a calculated fault time changes by a single time step which is given by

$$
\Delta_{\text {step }}=\left(d_{1}+\Delta_{\text {step }}\right)-d_{1}=\frac{1}{2}\left(l+u\left(\left(t_{1}+\frac{1}{S R}\right)-t_{2}\right)\right)-\frac{1}{2}\left(l+u\left(\left(t_{1)}-t_{2}\right)\right)\right.
$$

Where $\Delta_{\text {step }}$ is the step size, or minimum detectable change in meters, and $S R$ is the sampling rate in samples per second. This can be solved to find that $\Delta_{\text {step }}=\frac{u}{2(S R)}$

The factor of 2 is presented since a difference of one sample at one end of the transmission line only makes half the impact of one sample at each end in opposite directions. The value of $\Delta_{\text {step }}$ is used in determining the maximum accuracy available for any given transmission line and it is important for shorter transmission lines where the error per length of the transmission line can be significantly affected.

\subsection{Analysis Using the Magnetic Field}

As previously stated, the most obvious coordinate system to analyze a rotating magnetic field is a polar coordinate system, since any changes in the expected total magnetic field will be detected easily in this way. Four algorithms are presented in this section for the detection of faults while examining the system in polar 
coordinates. All of them involve detecting if the values of rho or theta have exceeded, or gone below a set of expected boundaries, or have made a significant and unexpected change.

Since each of these algorithms has a possibility of incorrectly detecting a fault, the results of these algorithms can be analyzed collectively to better determine whether or not a fault has truly occurred. Also, by taking the earliest fault detection times from each algorithm, the microprocessor which is performing this analysis will be able to determine actual fault detection times more correctly in order to perform the fault location more accurately. As a result, this combined analysis using all of these algorithms will provide a reduced number of "false alarms" as well as more accurate fault location.

\subsection{Analysis Algorithms}

The algorithms used in this analysis detect the steady-state magnetic field behavior and the determination of any deviation from it. The first algorithm estimates the ellipse formed by the magnetic field then detects any significant deviations from this locus. The next algorithm compares the present value of rho to the value detected a fraction of a cycle before it and determines if too significant of a change has taken place.

\subsection{The "Expected Ellipse" Algorithm}

Since the magnetic field will typically form an ellipse in steady state, the simplest way for a microprocessor is to determine if there is a fault to sense if the magnetic field intensities significantly change from the elliptical pattern. There are several ways to perform such an analysis. One way is to approximate the shape of the ellipse and determine. Once constant ellipse has been found, the instantaneous magnetic field value will deviate from that ellipse. Such a deviation from a constant ellipse is shown below in Figure 8 in order to analyze this rotating field in this way, which will be referred to as the "expected ellipse" algorithm, the following steps are performed:

1) The average maximum and minimum magnetic field intensities and any angle of rotation of the field are determined.

2) An ellipse approximating the rotating magnetic field is generated from this information.

3) Ellipses for the minimum and maximum allowable values of the magnetic field intensity based on allowable percentage deviation from the average must be created from the approximation; these are used to detect any sort of abnormal behavior.

Similar to the Cartesian coordinate ellipse discussed before, the polar coordinate ellipse which can be generated from the information about the maximum rho, minimum rho, and shifted angle is defined by

$$
\rho=\frac{\rho_{\max } \rho_{\min }}{\sqrt{\rho_{\max }^{2} \sin ^{2}\left(\theta-\theta_{\text {shift }}\right)+\rho_{\min }^{2} \cos ^{2}\left(\theta-\theta_{\text {shift }}\right)}}
$$

Where $\rho$ is the predicted value of rho for any given value of $\theta$ (based on the value of $\theta$ for any data point), $\rho_{\max }$ and $\rho_{\min }$ indicate maximum and minimum values of rho, while $\theta_{\text {shift }}$ reflect the rotational shift of the ellipse. Once this ellipse is constructed, it is used in determining allowable maximum and minimum magnetic fields. An example of elliptical rotating magnetic field with several boundaries of allowable values of rho for given values of theta is shown below in Figure 9; the actual magnetic field is shown in bold. While values of $\rho_{\text {max }}$ and $\rho_{\text {min }}$ are indicated, it should be noted that values of $\rho_{\max }$ and $\rho_{\min }$ occur at the points $180^{\circ}$ around the ellipse from the indicated points. The boundary which is significantly larger than the actual ellipse (the "maximum boundary") is used for determining if a fault definitely occurred in contrast to the possibility of a fault occurring. This method of analysis is effective in determining the maximum and minimum values of rho which can be determined accurately. However, if the steady-state currents are distorted, both the maximums and minimums and the angles at which they are detected could be affected. This effect can be reduced by adding an analog or digital filter to the detection device, but there is still a chance of the detection of maximums and minimums being slightly incorrect. Even if these values are close to real, there is a chance of either a "false alarm" or a fault not being detected with this algorithm alone. In order to decrease the number of these false positives, a different algorithm can be used in conjunction with the "expected ellipse" algorithm.

\subsection{The "Previous Value" Algorithm}

Since the values of rho in the polar coordinate system do not change significantly over a very short time step for a transmission line that is relatively well balanced, each value of rho can be compared against a value that occurred shortly before it to detect sudden changes. In a sense, this effectively compares the magnetic field 
against rotated and scaled versions of the same magnetic field. An example of an elliptical rotating magnetic field along with some boundaries generated for this "previous value algorithm" is shown in Figure 10. The "previous value" algorithm is especially useful in cases where the magnetic field is not exactly an ellipse and thus cannot be accurately monitored with the "expected ellipse" algorithm. The use of two detection algorithms in conjunction with each other can reduce incorrect fault detections. For example, if the system is fairly imbalanced, faults will be more likely to be incorrectly detected with the "previous value" algorithm, while the "expected ellipse" algorithm will not have as much of a problem with this. Similarly, if harmonics are seen by the sensors and are not properly filtered, the "expected ellipse" algorithm will be much more likely to detect a fault incorrectly while the "previous value" algorithm will not. A magnetic field with several unfiltered arbitrarily phase-shifted harmonics is presented below, while Figure 11 shows the same magnetic field with boundaries based on the "expected ellipse" algorithm. It is clear that the "expected ellipse" algorithm will incorrectly detect faults in this situation. Ideally, all of the harmonics would be filtered prior to analysis, but complete filtering would have negative impacts on the system in other ways including making faults harder to detect. As a result, it cannot be assumed that the magnetic field will be a perfect ellipse. Thus, the fault detection results of the "expected ellipse" algorithm are combined with the results of the "previous value" algorithm in order to better determine if a fault has truly occurred. This example reinforces the idea that performing an analysis of the magnetic field using multiple algorithms in conjunction with one another can reduce the number of incorrect fault detections if the results from each algorithm are compared against those from the other algorithms.

\section{Conclusion}

This paper described the theory and methods of traveling wave fault detection and location using magnetic field sensors. The concept of the magnetic field for a general and three phase system was explored. This was followed by a presentation of the magnetic fields for a variety of conductor configurations and sensor locations. The algorithms used in the magnetic field-based fault detection are then described. The magnetic field sensors are found to be effective in detecting faults conceptually. Additionally, the algorithms theoretically can provide accurate fault detection for common types of faults. All of these prove that the magnetic field sensor to be a viable tool for power transmission line fault detection. Eventually a prototype of the magnetic field-based fault detector could be built and field tested. This would require more development of the sensors as well as harmonic-filtering circuitry. a MATLAB code is used to plot the ellipses of magnetic field of a transmission line.

\section{References}

A. Elhaffar, M. Lehtonen. (2004). Traveling Waves Based Earth Fault Location in 400kV Transmission Network Using Single End Measurement, in Large Engineering Systems Conference on Power Engineering, pp. 53-56.

D. A. Tziouvaras, J. B. Roberts, G. Benmouyal. (2001). New Multi-Ended Fault Location Design for Two- or Three-Terminal Lines," in Developments in Power System Protection (IEE), Conference Publication No. 479, , pp.395-398.

H. Khorashadi-Zadeh, M. Sanaye-Pasand. (2006). Correction of saturated current transformers secondary current using ANNs, IEEE Trans. Power Delivery, 21, 1, pp. 73-79.

IEEE Power Engineering Society (PES). (2004). IEEE Guide for Determining. Fault Location on AC Transmission and Distribution Lines, IEEE Std. C37.114TM.

IEEE Power Systems Relaying Committee (PSRC). (1999). IEEE Guide for Protective Relay Applications to Transmission Lines, IEEE Std. C37.113-, pp. 31.

J. Jiang, Y. Lin, J. Yang, T. Too, C. Liu. (2000). An Adaptive PMU Based Fault Detection/Location Technique for Transmission Lines-Part II: PMU Implementation and Performance Evaluation, IEEE Transactions on Power Delivery, Vol. 15, No. 4, pp. 1136-1146.

K. Firouzjah, A. Sheikholeslami. (2009). A current independent method based on synchronized voltage measurement for fault location on transmission lines, Simulation Modelling Practice and Theory, 17, pp.692-707.

K. Zimmerman, D. Costello. (2005). Impedance-Based Fault Location Experience, 58th Annual Conference for Protective Relay Engineers,2005, pp. 211-226.

Lian B. and Salama M.M.A. (1994). An overview of digital fault location algorithms for power transmission lines using transient waveforms.-Electric Power Syst. Res., Vol. 29, No. 1, pp. 17-25.

M. Aurangzeb, P. A. Crossley, P. Gale. (2000). Fault Location on a Transmission Line Using High Frequency 
Travelling Waves Measured at a Single Line End," in Power Engineering Society Winter Meeting, Vol. 4, , pp. 2437-2442.

M. Sneddon, P. Gale. (1997). Fault Location on Transmission Lines, IEEE Colloquium on Operational Monitoring of Distribution and Transmission Systems, January, pp. 2/1-2/3.

M. Vintan. (2004). Fault Current Distribution Computation on Overhead Transmission Lines, in Proceedings of the Fifth International World Energy System Conference, vol. II, Oradea, Romania, pp. 273-279.

Tahar bouthiba. (2004), fault location in EHV transmission lines using artificial neural networks, Int. J. Appl. Math. Comput. Sci., 14, 1, pp .69-78.

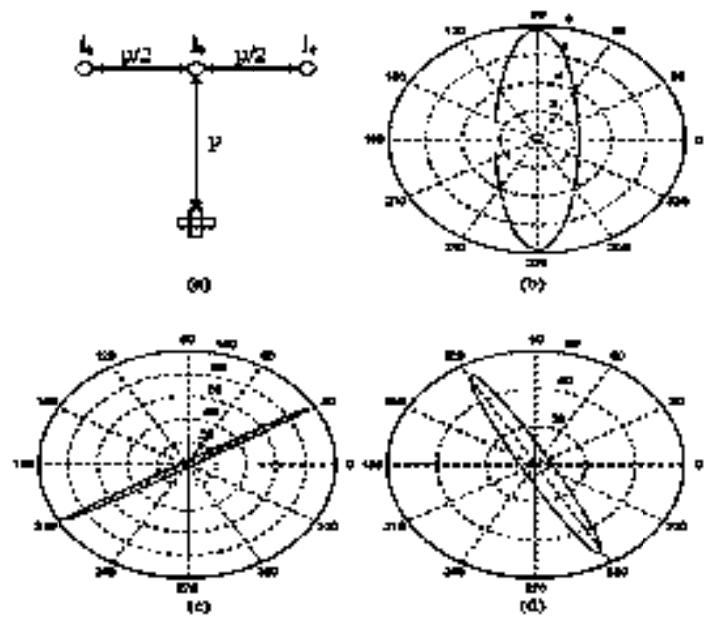

Figure 1. Horizontal conductor configuration and plots of its magnetic field

(a) - Conductor geometry; (b) - Magnetic field for balanced currents, $\mathrm{p}=2 \mathrm{~m}$;

(c) - Magnetic field for a line to ground fault on phase a, $\mathrm{p}=2 \mathrm{~m}$;

(d) - Magnetic field for line to line fault on phases a and $b, p=2 m$ 


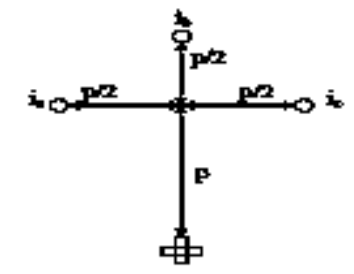

$\infty$
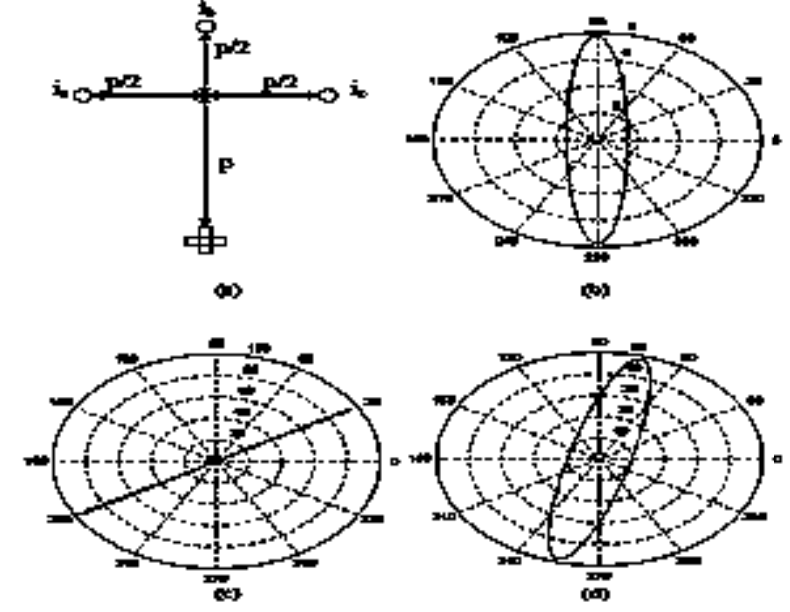

m

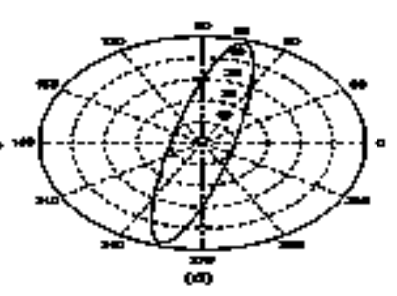

Figure 2. A delta conductor configuration and plots of its magnetic field (a) - Conductor geometry; (b) - Magnetic field for balanced currents, $\mathrm{p}=2 \mathrm{~m}$;

(c) - Magnetic field for a line to ground fault on phase a, $\mathrm{p}=2 \mathrm{~m}$;

(d) - Magnetic field for line to line fault on phases a and $b, p=2$

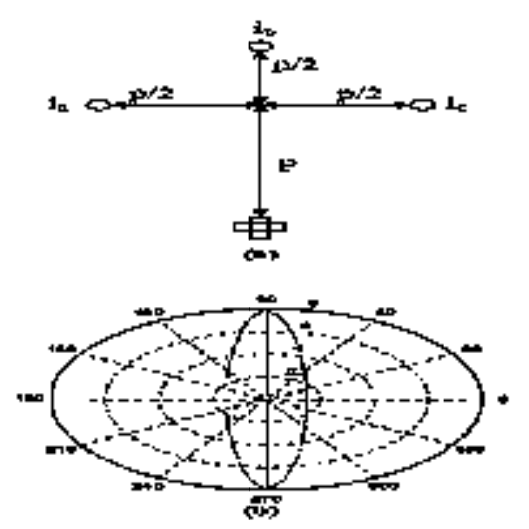

Figure 3. Delta conductor configuration and magnetic field with sensors under center phase

(a) - Conductor geometry; (b) - Magnetic field for balanced currents, $p=2 m$ 


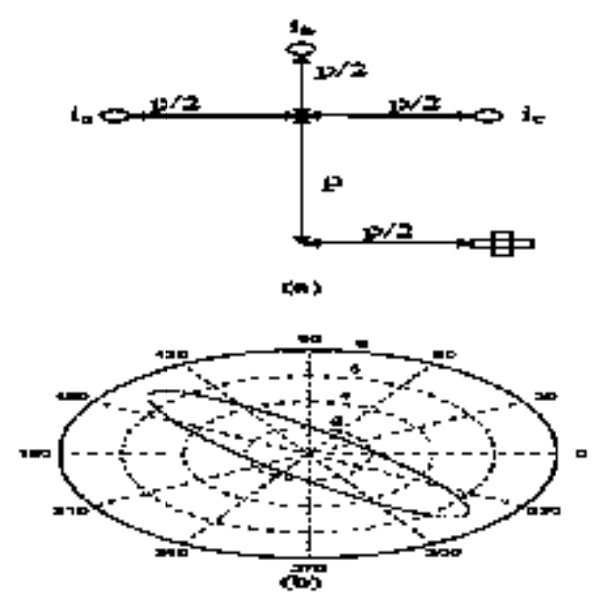

Figure 4. Delta conductor configuration and magnetic field with sensors shifted (a) - Conductor geometry; (b) - Magnetic field for balanced currents, $p=2 m$

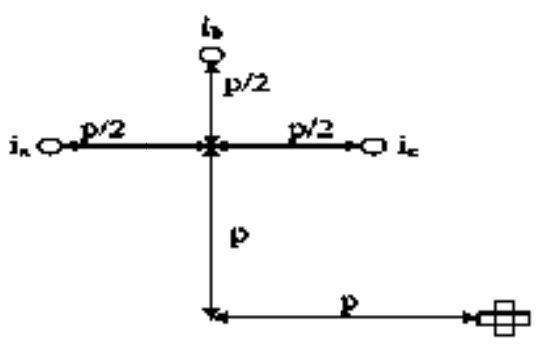

(a)

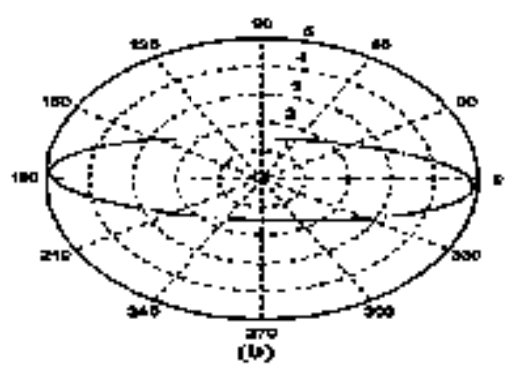

Figure 5. Delta conductor configuration and magnetic field with sensors shifted farther (a)-Conductor geometry; (b) - Magnetic field for balanced currents, $p=2 m$ 


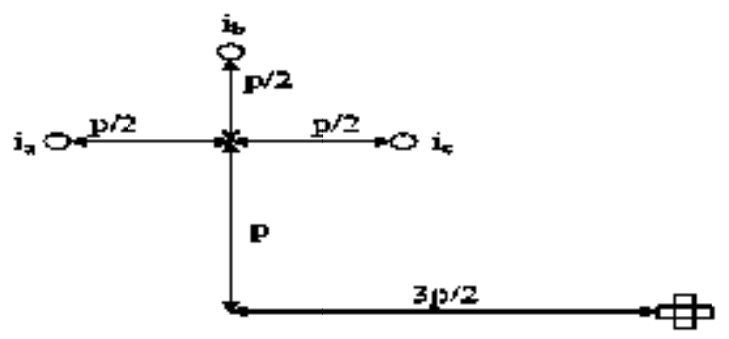

ips

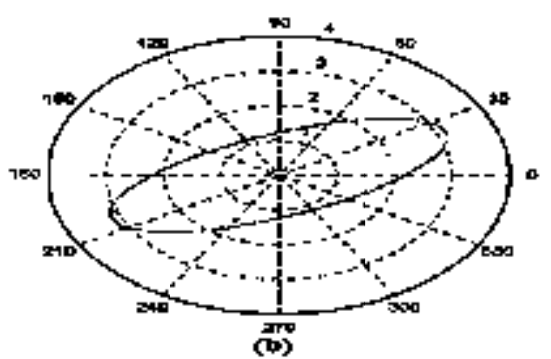

Figure 6. Delta conductor configuration and magnetic field with sensors shifted significantly (a) - Conductor geometry; (b) - Magnetic field for balanced currents, $p=2 m$

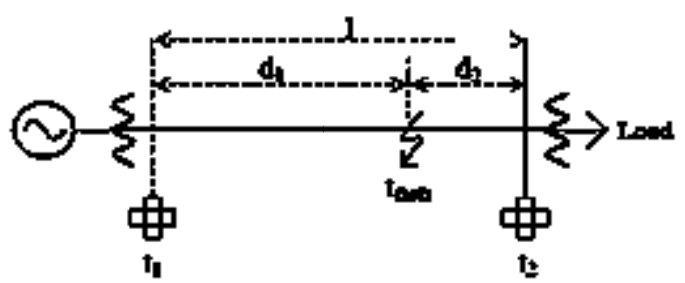

Figure 7. distances and times used in determining the fault location 


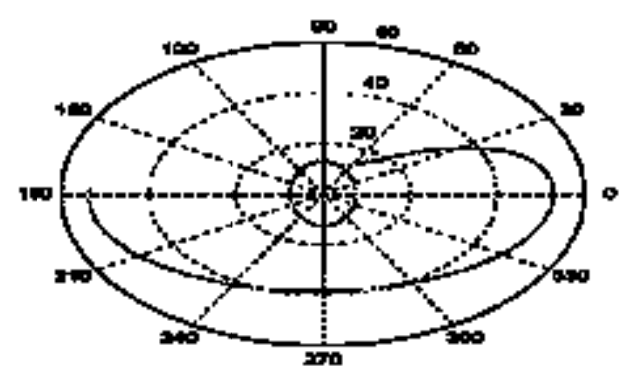

Figure 8. example of a deviation from the original ellipse

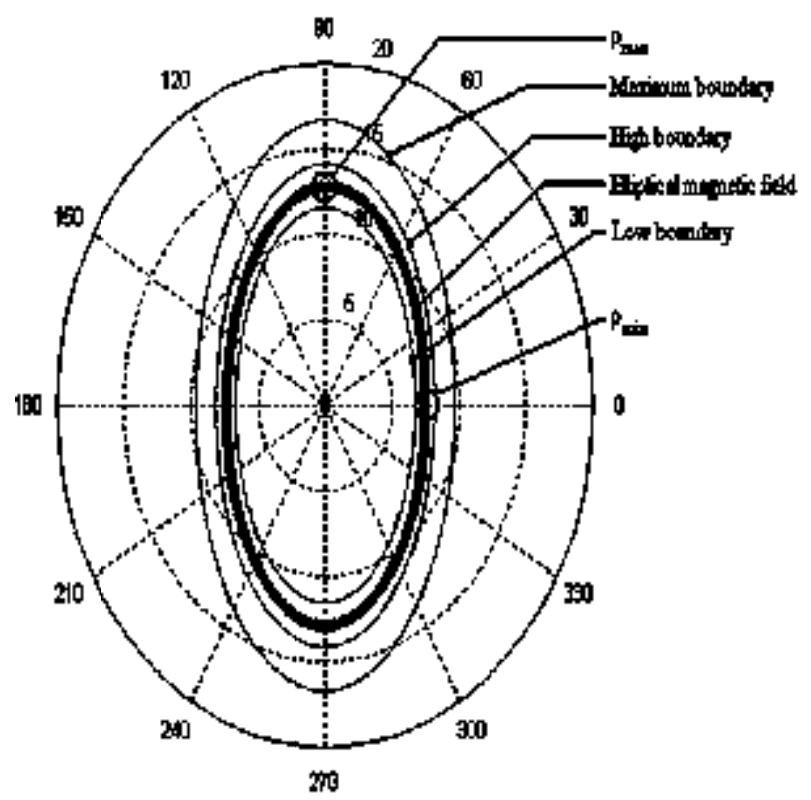

Figure 9. Elliptical rotating magnetic field with boundaries for the "expected ellipse" algorithm 


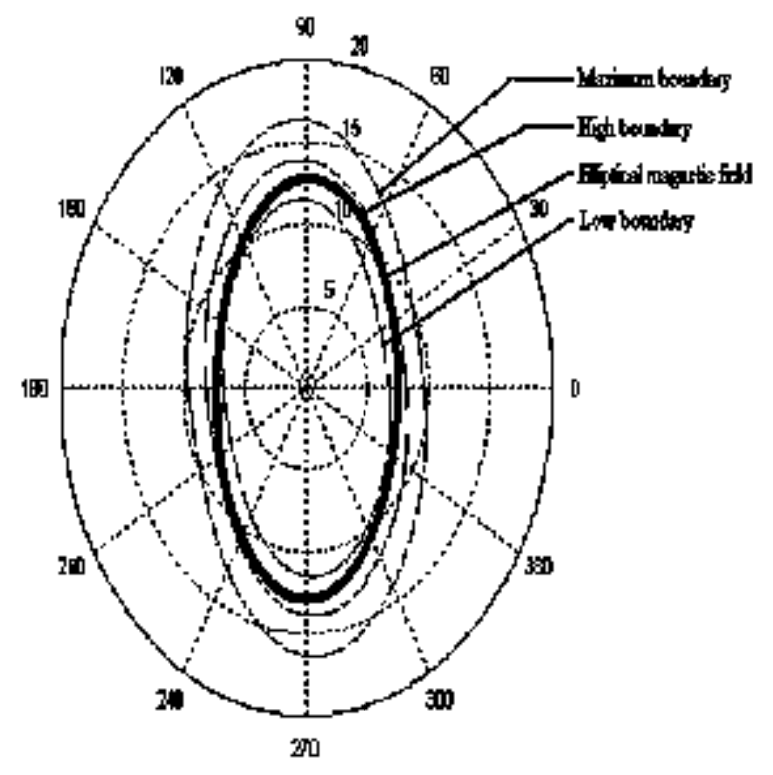

Figure 10. Elliptical rotating magnetic field with boundaries for the "previous value" algorithm

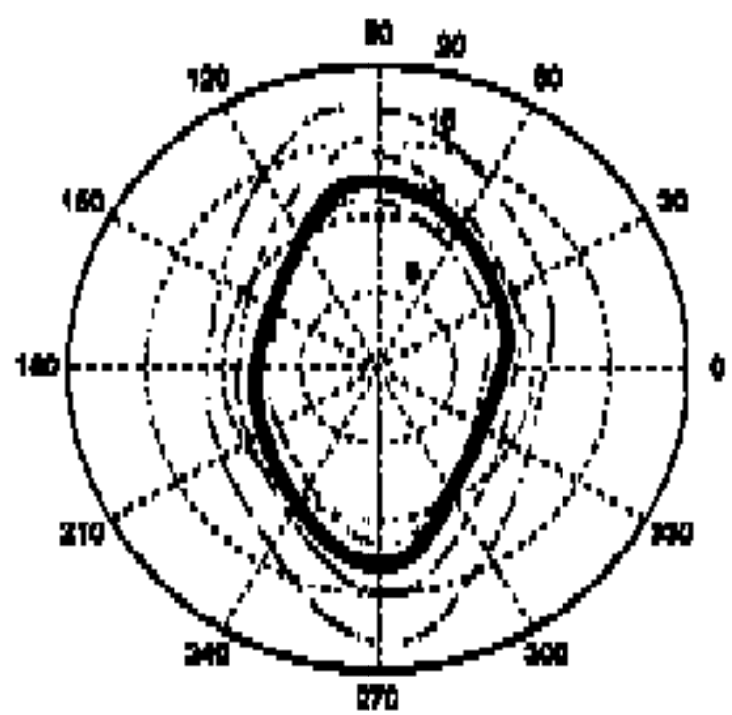

Figure 11. Magnetic field with harmonics, monitored by "previous value" algorithm 